\title{
Intoxicação por antibióticos ionóforos e leucoencefalomalácia concomitantes pelo consumo de ração comercial contaminada em equinos
}

\author{
Concurrent lonophore Poisoning and Leukoencephalomalacia by Consumption \\ of Contaminated Commercial Ration in Horses
}

\begin{abstract}
Mizael Machado', Larissa Rocha Andrade², Daniella Dianese Alves de Moraes³, Márcio Botelho de Castro', Antônio Raphael Teixeira Neto ${ }^{2}$, Kelly Moura Keller ${ }^{4}$, Benito Soto-Blanco ${ }^{5}$ \& Antônio Carlos Lopes Câmara ${ }^{2}$
\end{abstract}

Background: Equidae nutritional change increased the frequency of diseases due to inappropriate administration and storage of rations. Although there are reports of ionophore poisoning (IP) and leukoencephalomalacia (LEM) in equidae from Brazil, the concurrent occurrence of both diseases by the same contaminated commercial ration is unprecedented. Therefore, the present paper aims to describe the epidemiological, clinical, laboratorial and pathological findings of concurrent IP and LEM outbreaks in horses.

Cases: Eleven farmers from seven different locations in Distrito Federal, Midwestern Brazil, reported sudden clinical signs in 27 horses after the ingestion of commercial pelleted ration. During the farm visits, it was found that the ration brand and batches were identical, and macroscopic evaluation revealed no abnormalities. Eight horses were clinically evaluated and presented hyporexia, apathy, hypermetria, ataxia, dehydration, dyspnea, tongue hypotonia, muscle tremors, tachycardia, facial hypoalgesia, dysphagia, and sporadic or permanent recumbence. Laboratorial changes were restricted to creatine phosphokinase (mean: 1,573.4 $\pm 16.9 \mathrm{IU} / \mathrm{L}$ ) and gammaglutamyl transferase (mean: $34.85 \pm 29.14 \mathrm{IU} / \mathrm{L}$ ) serum increases. Pathological evaluation has performed in eight horses presenting pallor and whitish striations in the gluteal, longissimus dorsi, femoral quadriceps muscles and myocardium, varying from mild to moderate. One horse also showed a soft and yellowish focal area on the right temporal lobe white matter. Microscopically, alterations in skeletal and cardiac muscle tissues included striated muscle fibers and cardiomyocytes segmental necrosis, characterized by sarcoplasmic fragmentation with clusters of eosinophilic debris, cellular retraction and hypereosinophilia. Histological alterations in the central nervous system of one horse were characteristic of LEM. Ration samples analysis revealed the presence of salinomycin $(2,384.91 \pm 100.7 \mathrm{ppm})$, narasin $(144.15 \pm 7.75 \mathrm{ppm})$ and fumonisins $(0.87$ to $4.09 \mathrm{ppm})$. Eight hospitalized horses were submitted to clinical therapy (gastric lavage and application of activated charcoal; fluid-electrolyte imbalance correction, intravenous dimethylsulfoxide, tocopherol and selenium supplementation). Four (50\%) horses had permanent sequelae (muscle weakness and mild ataxia), two (25\%) recovered without sequelae, and two (25\%) were euthanized due to inability to stand and poor prognosis. Considering the total number of affected horses, mortality rate reached 59.25\% (16/27). Discussion: Usually ionophores are safely used in livestock and bird production at the recommended doses. However, horses are among the most susceptible species and do not tolerate any exposure to the substance. The outbreaks herein occurred due accidental ionophore contamination of commercial ration at the industry probably by manufacturing process technical failures. Similarly, fumonisins contamination may be caused by feedstock inadequate storage at the industry, or improper ration storage in the farms. The suspicion of two concurrent diseases (IP and LEM) in the horses of these outbreaks was confirmed through the association of epidemiology, clinical signs, pathological findings and measurement of ionophores and fumonisins in the commercial ration. We reiterate the difficulty in diagnosing associated clinical syndromes, especially when high-lethality diseases are involved.

Keywords: cardiopathy, fumonisins, malacia, narasin, salinomycin, toxic miopathy.

Descritores: cardiopatia, fumonisinas, malácia, miopatia tóxica, narasina, salinomicina. 


\section{INTRODUÇÃO}

A equideocultura é uma atividade em expansão, que cresce a cada ano e profissionalizou-se com o fortalecimento dos esportes equestres. Atualmente, estes animais são considerados atletas, necessitando de suplementação para melhor desenvolvimento das novas atividades. Tal mudança nutricional aumentou a frequência de enfermidades decorrentes da administração e armazenamento inadequado de rações [6]. Dentre elas, destacam-se enterites, cólicas, laminites e micotoxicoses, como a leucoencefalomalácia (LEM) [15]. Ainda, tais rações podem conter antibióticos ionóforos (AI), que são utilizados como coccidiostáticos, antimicrobianos e promotores do crescimento para muitas espécies animais $[4,13]$. Embora considerados seguros, quando usados para a espécie-alvo e dentro das doses recomendadas, equídeos são particularmente sensíveis a tais compostos [3,4,13,14].

Apesar de existirem relatos de intoxicação por AI $[1,3,12,14]$ e LEM $[6,8,12,18,19,21]$ em equídeos no Brasil, a ocorrência simultânea de ambas as enfermidades pelo fornecimento da mesma ração comercial contaminada é inédito. Diante do exposto, objetiva-se descrever os achados epidemiológicos, clínicos, laboratoriais e patológicos de surtos de intoxicação por AI e LEM concomitantes em equinos.

\section{CASOS}

Onze proprietários de sete diferentes localidades no Distrito Federal, Centro-Oeste do Brasil, notificaram a ocorrência de sinais clínicos súbitos em 27 equinos após a ingestão de ração comercial (Tabela 1). Obteve-se o histórico detalhado, durante visitas às propriedades, juntos aos proprietários ou médicos veterinários. Os equinos recebiam entre 3 e $8 \mathrm{~kg}$ de ração peletizada comercial, sendo que alguns animais rejeitaram espontaneamente a ração e outros comeram menos do que o habitual. Durante as visitas às propriedades, constatou-se que a marca e os lotes da ração eram idênticos, mas a avaliação macroscópica não revelou anormalidades perceptíveis. Todos os proprietários afirmaram que os primeiros sinais clínicos se iniciaram 6 a $12 \mathrm{~h}$ após a ingestão da ração, e incluíram mucosas congestas, sudorese, salivação, tremores musculares e claudicação. Nesta fase, alguns equinos foram tratados com antiinflamatórios não esteroidais devido à suspeita inicial de cólica, porém sem melhora clínica aparente. Com o passar das horas, o quadro se tornava mais severo e os sinais clínicos evoluíam para ataxia, quedas, decúbito lateral, e em alguns equinos, culminou com o óbito em até $24 \mathrm{~h}$.
Oito equinos foram encaminhados para atendimento hospitalar, e submetidos a exame clínico [20], que evidenciou hiporexia (8/8), apatia (6/8), hipermetria (6/8), ataxia (6/8), desidratação (5/8), dispneia (5/8), hipotonia lingual (5/8) (Figura 1), tremores musculares (5/8), taquicardia (4/8), hipoalgesia de face (3/8), disfagia (1/8), decúbito esporádico (2/8) e permanente (2/8). Amostras sanguíneas para realização de hemograma, determinação da proteína plasmática total, ureia, creatinina e dosagem de enzimas hepáticas (aspartato aminotransferase [AST], alanina aminotransferase [ALT] e glutamiltransferase [GGT]) e muscular (creatina fosfoquinase [CK]) foram coletadas. As alterações laboratoriais se restringiram a aumentos séricos das enzimas CK e GGT com variação de 388 a 4.809 UI/L (média: 1.573,4 \pm 16,9 UI/L; valores de referência: < 140 U/L) e 15 a 90 U/L (média: 34,85 \pm 29,14 UI/L; valores de referência: 0 - 11,8 U/L) [11], respectivamente. Com base nos achados epidemiológicos, clínicos e laboratoriais, suspeitou-se de intoxicação por AI. Entretanto, a marcante insuficiência de nervos cranianos, principalmente o V (trigêmeo), VII (facial) e XII (hipoglosso) pares, observada no exame neurológico serviram de base para a suspeita de envolvimento concomitante de LEM.

Todos os oito equinos internados foram submetidos a terapia clínica, que consistiu de lavagem gástrica com posterior aplicação de carvão ativado (Enterex ${ }^{\circledR 1}$ : 0,2 g.kg $^{-1}$, via oral, dose única), correção do desequilíbrio hídrico-eletrolítico, administração de dimetilsulfóxido (Dimesol ${ }^{\circledR 2}: 1$ g.kg-1 em solução a 10\%, s.i.d., via endovenosa, 2 dias), e suplementação com tocoferol e selênio (E-S-E Super ${ }^{\circledR 1}: 20$ g/animal/dia, uso contínuo). Destes, quatro (50\%) equinos tiveram sequelas permanentes (fraqueza muscular e discreta ataxia), dois $(25 \%)$ recuperaram-se sem sequelas, e dois (25\%) foram eutanasiados devido à incapacidade de assumir estação e prognóstico ruim. Levando em consideração o número total de equinos acometidos nos surtos, o índice de mortalidade alcançou 59,25\% (16/27) [Tabela 1].

Do total de 16 equinos mortos ou submetidos à eutanásia in extremis, oito (50\%) foram necropsiados (Equinos A-H). Fragmentos de músculos estriados esqueléticos (longíssimo dorsal, quadríceps femoral e glúteo), coração, pulmões, sistema nervoso central (SNC), fígado, rins, baço, linfonodos, estômago e intestinos foram fixados em formalina a $10 \%$, processados rotineiramente para histologia. Os achados macroscópicos consistiram de mucosas conjuntivais, oral e genitais acentuadamente 
congestas, palidez e estriações esbranquiçadas nas musculaturas da região glútea, longíssimo dorsal, quadríceps femoral e miocárdio, que variavam de discretas a moderadas. O Equino D apresentou ainda área focalmente extensa amolecida e amarelada na substância branca do lobo temporal direito. Fragmentos do SNC foram enviados para realização de imunofluorescência direta para diagnóstico de raiva, com resultado negativo.

Alterações histopatológicas foram observadas nos tecidos musculares esqueléticos e cardíaco (Tabela 2) e compreendiam principalmente necrose segmentar, multifocal a coalescente de fibras musculares estriadas e cardiomiócitos, caracterizada por fragmentação sarcoplasmática com aglomerados de detritos eosinofílicos (Figura 2A) e retração celular e hipereosinofilia (Figura 2B). Dois equinos (B e G) ainda apresentaram fibroplasia discreta multifocal no coração e musculatura esquelética, respectivamente. Constatou-se também edema moderado nas áreas de necrose da musculatura esquelética do Equino A. No Equino D observou-se malácia focalmente extensa da substância branca no lobo temporal direito, preenchida e circundada por moderada quantidade de células Gitter (Figura 3), discreta hemorragia e moderado edema perivascular.

Amostras das rações das propriedades foram coletadas de sacos em uso e lacrados, e encaminhadas para análise de multiresíduos pelo método de cromatografia líquida acoplada à espectrometria de massas sequencial no Departamento de Controle de Fármacos e Contaminantes em Produtos para Alimentação Animal e Medicamentos Veterinários, Laboratório Nacional Agropecuário do Rio Grande do Sul (LANAGRO/RS), que demonstrou a presença de 2.384,91 $\pm 100,70$ ppm de salinomicina e 144,15 \pm 7,75 ppm de narasina. Amostras das mesmas rações também foram enviadas para detecção e quantificação de fumonisinas através de ELISA no Laboratório de Micologia e Micotoxinas, Universidade Federal de Minas Gerais, detectando nível total de fumonisinas variando de 0,87 a 4,09 ppm (média= 2,82 ppm).

\section{DISCUSSÃO}

Os AI geralmente são seguros e utilizados na produção de ruminantes e aves nas doses recomendadas. Entretanto, os equinos estão entre às espécies mais sensíveis à intoxicação e não toleram exposição à substância [3,4,14]. Os surtos aqui descritos ocorreram pela contaminação acidental da ração na indústria por AI, devido a falhas técnicas no processo de fabricação.
De forma semelhante, a contaminação conjunta com fumonisinas pode ser decorrente de falhas no controle de qualidade ou armazenamento inadequado da matéria prima na indústria, ou ainda, armazenamento impróprio nas respectivas propriedades, agravando os sinais clínicos e prognóstico devido à associação da LEM com os efeitos tóxicos dos AI.

Os sinais clínicos observados são semelhantes aos descritos em surtos anteriores de intoxicação por AI $[1,2,14]$. Entretanto a associação da epidemiologia com os achados de insuficiência de nervos cranianos em 62,5\% (5/8) dos equinos examinados, levantaram a suspeita de LEM concomitante. Acredita-se que o fato de alguns equinos rejeitarem espontaneamente ou ingerirem menores quantidades da ração associado a rápida terapia instituída influenciaram diretamente a absorção das fumonisinas, e, consequentemente a severidade dos sinais clínicos neurológicos; que são principalmente de origem cerebral ou do tronco encefálico $[6,10,15]$. Esta hipótese também explica a variação no tempo de evolução clínica e severidade das lesões musculares e cardíacas nos equinos intoxicados por AI.

As alterações laboratoriais, principalmente as dosagens de enzimas musculares, apresentaram-se diretamente correlacionadas a severidade dos sinais clínicos [2], onde equinos com menores dosagens destas enzimas possuíam sintomatologia mais branda, e, consequentemente, melhor prognóstico. Naqueles equinos submetidos a eutanásia, as altas dosagens das enzimas musculares também tiveram correlação direta com o grau de lesões histológicas [14]. As elevações da atividade sérica da enzima GGT em alguns equinos pode ser correlacionada a hepatotoxicidade comprovada das fumonisinas [6].

Com relação aos limites máximos admissíveis para esta classe de micotoxinas, não há regulamentação específica, talvez justificada por sua descoberta tardia. Em 2000, o Food and Drug Administration (FDA) estabeleceu como normativa o limite de $5 \mathrm{ppm}$ de fumonisinas na alimentação de equídeos sem, contudo, ultrapassar $20 \%$ do peso da dieta seca. Posteriormente, estudo realizado pela Food and Agriculture Organization of the United Nations (FAO) mostrava que, em 2003, apenas seis países já haviam fixado seus valores limites, sendo em quatro países até 1000 ppb ou 1 ppm (equivalente a $1 \mu \mathrm{g} / \mathrm{g}$ ); um país até $2 \mathrm{ppm}$; e um país até $3 \mathrm{ppm}$ [9]. No Brasil, ainda não há legislação específica, apesar da importância destas micotoxinas no cultivo do milho. Assim, os níveis de concentração de até 4,82 ppm de fu- 
Tabela 1. Epidemiologia e distribuição dos casos de intoxicação por antibióticos ionóforos e leucoencefalomalácia em equinos no Distrito Federal, Centro-Oeste do Brasil.

\begin{tabular}{cccccc}
\hline Propriedade & Localidade & Equinos mortos & \multicolumn{2}{c}{ Equinos vivos } & \multirow{2}{*}{ Total } \\
\cline { 3 - 4 } & & & Com sequelas & Sem sequelas n & \\
\hline 1 & Riacho Fundo & $3^{*}$ & 4 & 0 & 7 \\
2 & Planaltina & 2 & 0 & 1 & 3 \\
3 & Samambaia & 0 & 1 & 0 & 1 \\
4 & Gama & 3 & 0 & 1 & 4 \\
5 & Samambaia & 1 & 0 & 0 & 1 \\
6 & Samambaia & 1 & 1 & 0 & 3 \\
7 & Ceilândia & 2 & 0 & 1 & 1 \\
8 & Gama & 0 & 0 & 1 & 1 \\
9 & Sobradinho & 1 & 0 & 0 & 2 \\
10 & Guará & 1 & 0 & 0 & 2 \\
11 & Gama & 2 & 0 & $5(18,5 \%)$ & $27(100 \%)$ \\
\hline
\end{tabular}

"Um dos equinos apresentou lesões histológicas de leucoencefalomalácia (Equino D).

Tabela 2. Principais alterações histológicas no músculo esquelético e cardíaco de equinos intoxicados por antibióticos ionóforos no Distrito Federal.

\begin{tabular}{ccccccccccc}
\hline EQ & \multicolumn{2}{c}{ HDCS } & \multicolumn{2}{c}{ FRAG } & \multicolumn{2}{c}{ REGENERAÇÃO } & \multicolumn{2}{c}{ CONGESTÃO } & \multicolumn{2}{c}{ INFI } \\
\hline & ME & CO & ME & CO & ME & CO & ME & CO & ME & CO \\
A & ++ & ++ & +++ & - & - & - & - & + & $+(\mathrm{LH})$ & - \\
$\mathrm{B}$ & + & ++ & + & + & ++ & - & - & + & - & $+(\mathrm{HI})$ \\
$\mathrm{C}$ & + & - & - & - & - & - & - & - & $+(\mathrm{LH})$ & - \\
$\mathrm{D}$ & - & + & + & - & + & - & + & + & - & - \\
$\mathrm{E}$ & + & $\mathrm{NA}$ & - & $\mathrm{NA}$ & - & $\mathrm{NA}$ & + & $\mathrm{NA}$ & $+(\mathrm{NE})$ & $\mathrm{NA}$ \\
$\mathrm{F}$ & ++ & + & ++ & + & ++ & - & - & + & - & $+(\mathrm{NE})$ \\
$\mathrm{G}$ & + & - & ++ & - & +++ & - & - & - & $+(\mathrm{LH})$ & - \\
$\mathrm{H}$ & + & - & + & - & - & - & - & + & - & - \\
\hline
\end{tabular}

EQ: equino avaliado; ME: músculo esquelético; CO: coração. HDCS: hialinização e desaparecimento das estriações das fibras musculares; FRAG: fragmentação das fibras musculares; INFI: Infiltrado inflamatório (tipo: LH= linfohistiocítico; HI= histiocítico; NE= neutrofílico). NA: não avaliado. Grau da lesão: - ausente; + discreto; + + moderado; ++ + acentuado. * Adicionalmente, apresentava lesões de leucoencefalomalácia no sistema nervoso central.

monisinas nas amostras de ração associadas aos achados patológicos de LEM tornam consistente o diagnóstico. Existe certa divergência quanto aos níveis passíveis de toxicose, com alguns autores afirmando que alimentos com concentração menor que 10 ppm são seguros para equídeos [10,17]. Enquanto outros verificaram surtos com alta mortalidade de equinos consumindo ração com concentrações menores que 5 ppm $[18,19]$. Recentemente, dois surtos com mortalidade de 13 equinos ocorreram devido a ingestão de feno com concentração de fumonisinas variando de 0,02 a 0,12 ppm [21]. Ao que parece, alimentos com concentrações de fumonisinas até
10 ppm já são suficientemente nocivos, determinando risco de óbito se consumidas por equídeos [19].

As alterações patológicas associadas a intoxicação por AI caracterizaram-se por lesões degenerativo-necróticas nos grandes grupos musculares estriados esqueléticos, ainda mais intensas que as observadas no miocárdio [13]. De modo geral, estas intoxicações produzem lesões semelhantes, predominantemente nestes grandes grupos musculares em equinos $[3,4,14]$, assim como regiões dorsolombares, glúteos e ombros em ovinos [5]. As lesões teciduais observadas ocorrem principalmente devido à inibição do metabolismo energético devido a alteração do $\mathrm{pH}$ 


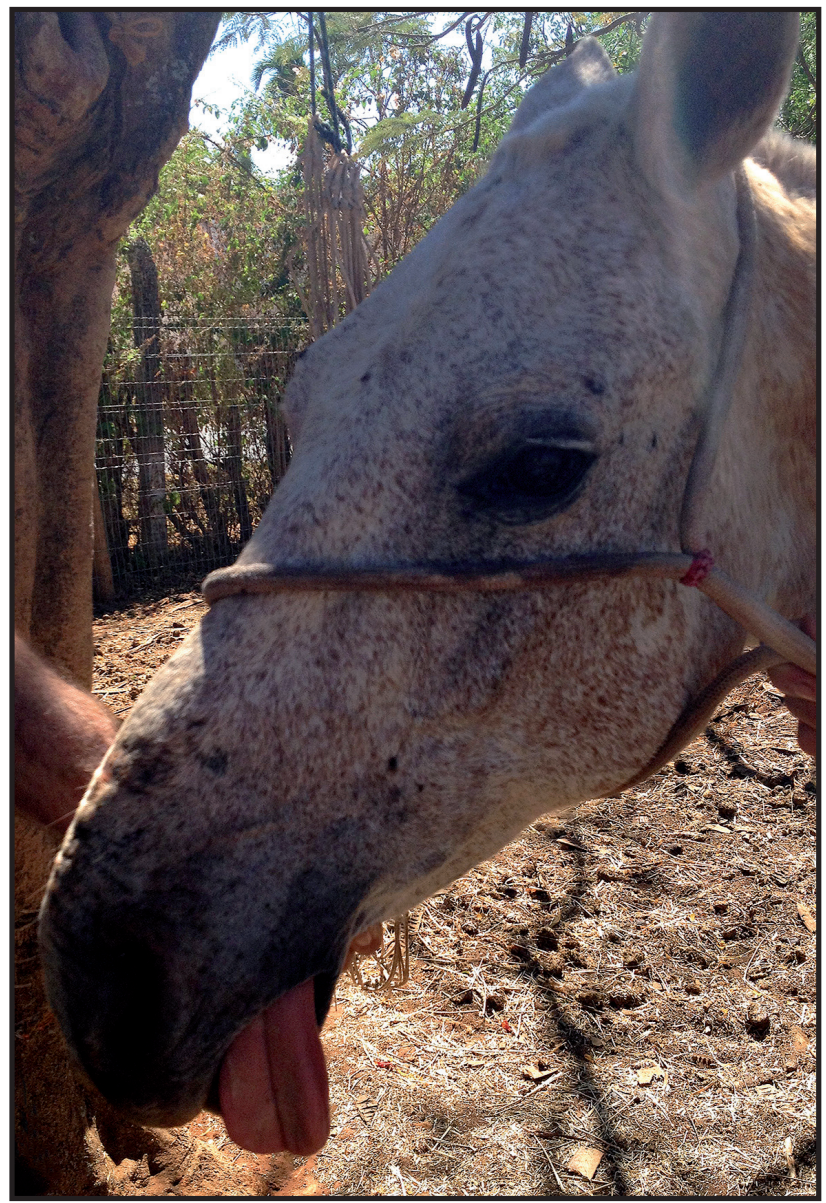

Figura 1. Equino apresentando apatia e hipotonia de língua.

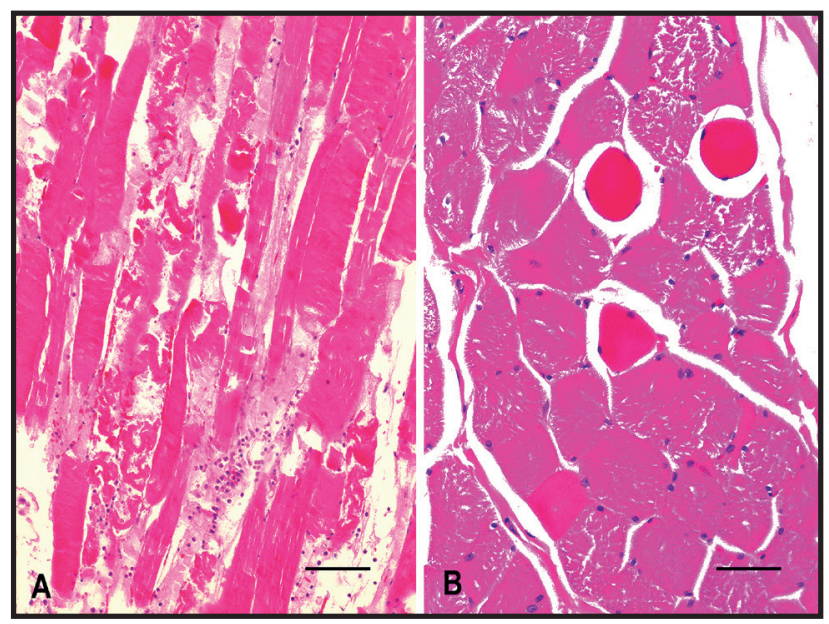

Figura 2. Equino, músculo estriado esquelético. A- Secção longitudinal evidenciando fragmentação sarcoplasmática e agregados eosinofílicos [HE; Barra $=100 \mu \mathrm{m}]$. B- Secção transversal demonstrando retração citoplasmática multifocal com intensa hipereosinofilia [HE; Barra $=50 \mu \mathrm{m}$ ].

entre as membranas e organelas e no transporte iônico de $\mathrm{Na}^{+}, \mathrm{K}^{+}$e $\mathrm{Ca}^{++}$. Isso promove o aumento do influxo celular de sódio e água, mobilização para citoplasma dos depósitos intracelulares de cálcio, edema das organelas e morte celular $[13,16]$. Enquanto as lesões mitocondriais decorrem da perda do controle do metabolismo osmótico, aumento na permeabilidade das

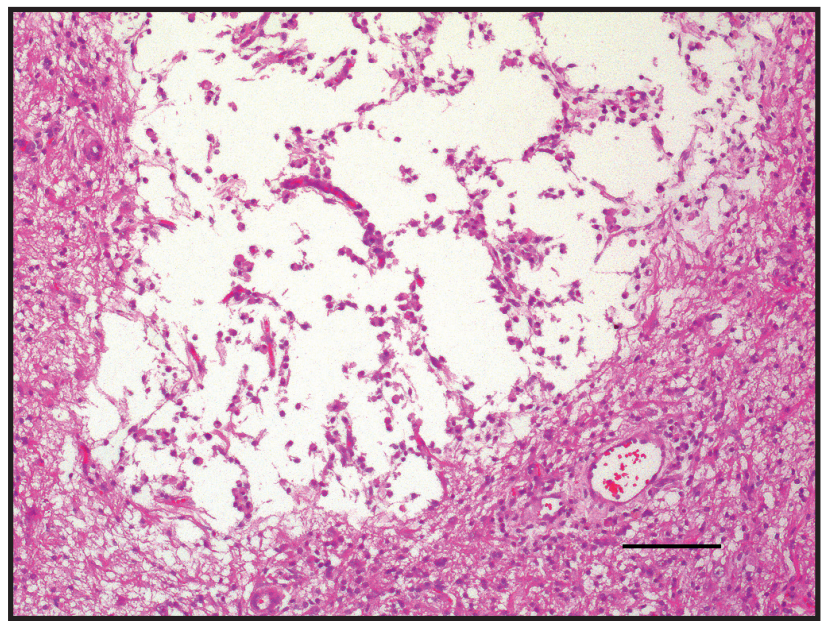

Figura 3. Equino, lobo encefálico temporal direito. Malácia e cavitação do parênquima encefálico com presença moderada de células Gitter [HE; Barra $=100 \mu \mathrm{m}]$.

membranas celulares e retículo sarcoplasmático, e provável alteração no metabolismo lipídico [7].

A contaminação concomitante da ração comercial com fumonisinas pode ter sido a responsável pela insuficiência de nervos cranianos observada no exame neurológico, além de causar lesões macroscópicas compatíveis com LEM em um cavalo (Equino D). Nessas áreas de malácia, as principais alterações histológicas consistiram de hemorragia, edema, dissolução do parênquima e cavitação; consideradas lesões características da doença em equídeos $[6,8,10,15,19,21]$. Tais lesões resultam da inibição da enzima ceramida sintase, levando a distúrbios na biossíntese de esfingolipídios. A inibição desta via metabólica resulta em depleção do complexo esfingolipídio, aumento intracelular da concentração de esfinganina e esfingosina livre, sendo o acúmulo destas bases esfingóides a causa primária da toxicidade das fumonisinas [22].

A suspeita de duas enfermidades (intoxicação por AI e LEM) concomitantes nos equinos destes surtos foram confirmadas através da associação da epidemiologia, sinais clínicos, achados patológicos e mensuração dos AI e fumonisinas na ração comercial. Reitera-se a dificuldade no diagnóstico de síndromes clínicas associadas, principalmente quando envolvidas doenças de alta letalidade.

\section{MANUFACTURERS}

${ }^{1}$ Vetnil Industria e Comércio de Produtos Veterinários Ltda. São Paulo, SP, Brazil.

${ }^{2}$ Hertape Calier Saúde Animal S.A. Juatuba, MG, Brazil.

Declaration of interest. The authors report no conflicts of interest. The authors alone are responsible for the content and writing of the paper. 


\section{REFERENCES}

1 Alberton L.R., Pachaly J.R., Belletini S.T. \& Giovanelli D.F. 2011. Intoxicação por salinomicina em equinos - estudo de um surto em 39 animais. Arquivos de Ciências Veterinárias e Zoologia UNIPAR. 14(1): 29-32.

2 Bautista A.C., Tahara J., Mete A., Gaskill C.L., Bryant U.K. \& Puschner B. 2014. Diagnostic value of tissue monensin concentrations in horses following toxicosis. Journal of Veterinary Diagnostic Investigation. 26(3): 423-427.

3 Bezerra Jr. P.S., Ilha M.R.S, Langohr I.M. \& Barros C.S.L. 2000. Intoxicação experimental por monensina em equinos. Pesquisa Veterinária Brasileira. 20(3):102-108.

4 Borges A.S., Silva D.P.G, Gonçalves R.C., Kuchembuck M.R.G., Chiacchio S.B., Mendes L.C.N. \& Bandarra E.P. 2001. Ionóforos e equinos: uma combinação fatal. Revista de Educação Continuada CRMV-SP. 4(2): 33-40.

5 Bourque J.G., Smart M. \& Wobeser G. 1986. Monensin toxicity in lambs. Canadian Veterinary Journal. 27(10): 397-399.

6 Câmara A.C.L., Afonso J.A.B., Riet-Correa F., Dantas A.F.M., Mendonça C.L., Costa N.A., Dantas A.C., Costa Neto H.A., Campos A.G.S.S. \& Souza M.I. 2008. Leucoencefalomalácia em equídeos no Estado de Pernambuco. Ciência Animal Brasileira. 9(2): 470-479.

7 Confer A.W., Reavis D.U. \& Panciera R.J. 1983. Light and electron microscopic changes in cardiac and skeletal muscle of sheep with experimental monensin toxicosis. Veterinary Pathology. 20(5): 590-602.

8 Del Fava C., Lara M.C.C.S.H., Villalobos E.M.C., Nassar A.F.C., Cabral A.D., Torelli C.S., Cunha M.S. \& Cunha E.M.S. 2010. Ocorrência de leucoencefalomalácia (LEME) em equídeos no Estado de São Paulo, Brasil: achados anatomopatológicos. Brazilian Journal of Veterinary Research and Animal Science. 47(6): 488-494.

9 FAO. 2004. Worldwide regulations for mycotoxins in food and feed in 2003. FAO Food and Nutrition Paper 81. Roma: FAO, 165p.

10 Giannitti F., Diab S.S., Pacin A. M., Barrandeguy M., Larrere C., Ortega J. \& Uzal F.A. 2011. Equine leukoencephalomalacia (ELEM) due to fumonisins B1 and B2 in Argentina. Pesquisa Veterinária Brasileira. 31(5): 407-412.

11 González F.H.D. \& Silva S.C. 2006. Introdução à Bioquímica Clínica Veterinária. 2.ed. Porto Alegre: Editora da UFRGS, 358p.

12 Marcolongo-Pereira C., Estima-Silva P., Soares M.P., Sallis E.S.V., Grecco F.B., Fernandes C.G., Raffi M.B. \& Schild A.L. 2014. Doenças de equinos na região Sul do Rio Grande do Sul. Pesquisa Veterinária Brasileira. 34(3): 205-210.

13 Nogueira V.A., França T.N. \& Peixoto P.V. 2009. Intoxicação por antibióticos ionóforos em animais. Pesquisa Veterinária Brasileira. 29(3): 191-197.

14 Pavarini S.P., Wouters F., Bandarra P.M., Souza F.S., Dalto A.G.C., Gomes D.C., Cruz C.E.F. \& Driemeier D. 2011. Surto de intoxicação por monensina em avestruzes e equinos no Sul do Brasil. Pesquisa Veterinária Brasileira. 31(10): 844-850.

15 Riet-Correa F., Rivero R., Odriozola E., Adrien M.L., Medeiros R.M. \& Schild A.L. 2013. Mycotoxicoses of ruminants and horses. Journal of Veterinary Diagnostic Investigation. 25(6): 692-708.

16 Roder J.D. 2011. Ionophore toxicity and tolerance. Veterinary Clinics of North America: Food Animal Practice. 27(2): 305-314.

17 Ross P.F., Rice L.G., Reagor J.C., Osweiler G.D., Wilson T.M., Nelson H.A., Owens D.L., Plattner R.D., Harlin K.A., Richard J.L., Colvin B.M. \& Banton M.I. 1991. Fumonisin B1 concentrations in feed from 45 confirmed equine leukoencephalomalacia cases. Journal of Veterinary Diagnostic Investigation. 3(3): 238-241.

18 Salles-Gomes T.L., Almeida P.E., Moreira M., Canola J.C., Canola P.A. \& Souza H.A. 2003. Surto de leucoencefalomalácia equina provocada por ração comercial com concentração de fumonisina inferior a 10 ppm. ARS Veterinária. 19(3): 267-271.

19 Santos C.E.P., Souto F.S.M., Santurio J.M. \& Marques L.C. 2013. Leucoencefalomalácia em equídeos da região Leste de Mato Grosso. Acta Scientiae Veterinariae. 41: 1119.

20 Spiers V.C. 1999. Exame clínico dos equinos. Porto Alegre: Artmed, 366p.

21 Vendruscolo C.P., Frias N.C., Carvalho C.B., Sá L.R.M., Belli C.B. \& Baccarin R.Y.A. 2016. Leukoencephalomalacia outbreak in horses due to consumption of contaminated hay. Journal of Veterinary Internal Medicine. 30(6): 1879-1881.

22 Zitomer N.C., Mitchell T., Voss, K.A., Bondy G.S., Pruett S.T., Garnier-Amblard E.C., Liebeskind L.S., Park H., Wang E., Sullards M.C., Merrill Jr. A.H. \& Riley R.T. 2009. Ceramide synthase inhibition by fumonisin $\mathrm{B}_{1}$ causes accumulation of 1-deoxysphinganine. Journal of Biological Chemistry. 284(8): 4786-4795. 